\title{
DTW and Voting-Based Lip Print Recognition System
}

\author{
Piotr Porwik and Tomasz Orczyk \\ Department of Computer Systems, University of Silesia, Katowice, Poland \\ \{piotr.porwik, tomasz.orczyk\}@us.edu.pl
}

\begin{abstract}
This paper presents a method of lip print comparison and recognition. In the first stage the appropriate lip print features are extracted. Lip prints can be captured by police departments. Traces from lips may also found at a crime scene. The approach uses the well known DTW algorithm and Copeland vote counting method. Tests were conducted on 120 lip print images. The results obtained are very promising and suggest that the proposed recognition method can be introduced into professional forensic identification systems.
\end{abstract}

Keywords: Lip print, matching, voting, image pre-processing, DTW, Copeland's voting method.

\section{$1 \quad$ Introduction}

Today we now know that not only fingerprints, footprints or pistol bullets with their ballistic traits, but also hair, voice, blood and the fibers from clothes can be treated as criminal identifiers. Some of identifiers mentioned above are captured by police technicians directly from crime scenes. Latent lip prints can be also successfully collected $[9,10,11,13]$. If properly captured and examined, the material left at a crime scene can contain useful data leading to personal identification. The factual materials collected can be successfully analyzed and recognized by the police or by medical professionals. Last year, a new type of identification trait was included in the practical repertoire of the police and the judiciary: the lip print. Lip prints are the impressions of human lips left on objects such as drinking glasses, cigarettes, drink containers and aluminum foils. The serious study of human lips as a means of personal identification was publicized in the 1970's by two Japanese scientists - Yasuo Tsuchihasi and Kazuo Suzuki $[2,3,4]$. The forensic investigation techniques in which human lip prints are analyzed are called a cheiloscopy $[1,3]$. The area of interest focused on by cheiloscopy is the system of furrows (lines, bifurcations bridges, dots, crossings and other marks) on the red part of human lips $[1,2,3]$. The uniqueness of lip prints makes cheiloscopy especially effective whenever appropriate evidence, such as lipstick blot marks, cups, glasses or even envelopes, is discovered at a crime scene. Even though lip print analysis is not substantially developed, this new form of identification is slowly becoming accepted and being introduced into practice all over the world. Nowadays, technique of lip print image analysis can be used for human identification, for example when a post mortem identification is needed [10]. It should be emphasized that cheiloscopy has already been successfully used as an evidence in lawsuits $[12,13]$. Unfortunately, use of the lip prints in criminal cases is often limited because

A. Cortesi et al. (Eds.): CISIM 2012, LNCS 7564, pp. 191-202, 2012.

(C) IFIP International Federation for Information Processing 2012 
the credibility of lip prints has not been firmly established in many courts. It should be mentioned here that a literature review shows that there are already a small number of works in which lip print technology (acquisition, automatic capture, analysis and recognition) have been mathematically described or practically utilized. Lip prints of a person are unique and are quite similar to finger imprints. Additionally, similar to fingerprint patterns, lip prints possess the following specific properties: permanence, indestructibility and uniqueness [1,2,3]. Lip prints are determined by one's genotype, and are therefore unique and stable throughout the life of a human being. In the solution preferred by forensic laboratories, lip imprints are captured using special police materials (white paper, a special cream and a magnetic powder). This technique is also preferred by police researchers. Here, in the first step, the lip print is rendered onto a durable surface using a fingerprint powder (a different type of powder can be used depending on the surface type). Special backing tapes or specialized papers (cards) can be used as a background surface. The image, thus developed, is then converted into a digital image by a scanner. This is the method of capture that we utilized during our investigations, as it allows for the lip images to capture more precise textures. These techniques have been described in a number of papers [2, 6-13]. The recognition results reported in the aforementioned papers have now been significantly improved.

\section{$2 \quad$ Lip Print Pre-processing}

For the proper preservation of evidence, lip imprints should be carefully captured and then copied to appropriate materials by means of a special cream and a magnetic powder. The process of capturing an image of the lips starts from one corner of the mouth and ends at the opposite site of the mouth, thus all the details of the lips being copied onto a surface. In successful prints, the lines on the lips must be recognizable, not smudged, neither too light nor too dark, and the entire upper and lower lip must be visible. In the next step, this trace needs to be digitalized because a digital lip print preparation is absolutely necessary for the subsequent techniques. Lip imprints often have many artefacts: fragments of skin, hairs and others, all of which generate unwanted noise. For this reason, the lip print image, $I$, should be enhanced and all of its artefacts removed. Many lip print feature extraction algorithms have been reported in the literature [1, 6-13]. In this paper, a new approach to lip print extraction and analysis is presented. The proposed method includes introduction of a sequence involving image normalization, lip pattern identification and feature extraction. The normalization procedure allows for the standardization of lip print images; it involves a horizontal alignment and then the separation of the image into the upper and lower lip. Lip pattern extraction separates the furrows from their background, thus forming the lip pattern. The main goal of the feature extraction is to convert the lip pattern image into projections (specialized histograms) which can then be compared using the DTW algorithm [5].

\subsection{Image Normalization}

In the first stage, after obtaining the original lip image $I$ of dimension $M \times N$, its histogram is stretched, thus a new image, $I^{\text {new }}$, is obtained. 
Let $I=\left\{p_{i}\right\}_{i=1}^{M \cdot N}$ be a set of pixel values $p_{i}$ which form the image $I$, and $p_{i} \equiv I(x, y) \in\{0, \ldots, L\}$. Given this, the histogram stretching operation is performed via a simple mathematical operation:

$$
I^{\text {new }}(x, y)=\frac{255}{\max -\min }(I(x, y)-\min ),
$$

where:

$\min =$ minimum value of the elements in the set $I$,

$\max =$ maximum value of the elements in the set $I$.

After stretching all source images are gray-scale images, black pixels have assigned a value of 0 and white pixels - a value of 255. Other pixels take intermediate values. The process of determining the lip area in a lip print image consists of several steps (Fig. 1). In the first step, the background of the original image should be removed. This can be performed by the following operation:

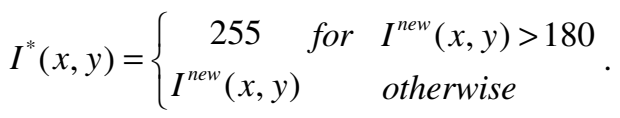

So pixels with a value greater than a threshold level (180) are converted into the colour white, while the remaining pixel values remain unchanged (Fig.1a). Next, a median filter with a $7 \times 7$ mask is used to blur the image $I^{*}$ (Fig.1b). The median filter has been chosen as it is much better at preserving sharp edges than a mean filter. This blurring operation forms the image $I^{* *}$. In the last step, a binary image is prepared. The process of forming a binary image is:

$$
I_{B I N}(x, y)=\left\{\begin{array}{ll}
0 & \text { for } I^{* *}(x, y)=255 \\
1 & \text { otherwise }
\end{array} .\right.
$$

The final, binary image is shown as Fig. 1c.
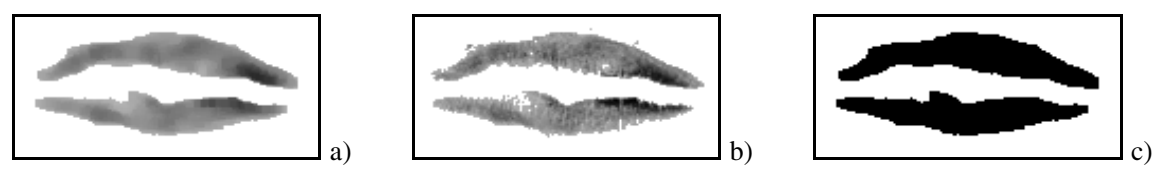

Fig. 1. Steps of lip area detection: a) the image after evaluating pixels against a threshold, b) after blurring, c) the detected lip area

The upper and lower lip separation is determined by a curve that runs through the centre of the space between the lips (Fig. 2a). This designated curve divides the region of interest of the lip print area into an upper and a lower lip.

A straight line equation can be established by means of a linear regression. It should be noticed that the normalized image presented in Fig. $2 b$ has been obtained from a high quality lip print. In practice, images captured can be of a poor quality - 
especially when the image has been captured as trace directly from a crime scene. For such images some intractable artefacts of the background may be difficult to eliminate. This means that the image cropping can not be performed perfectly. This can be seen in Fig. 3 in which normalized images are presented. The first image (Fig. 3a) is a good quality image, is perfectly normalized and thus leads to a properly cropped imprint. This image could be, for example, captured in a police department under ideal conditions.
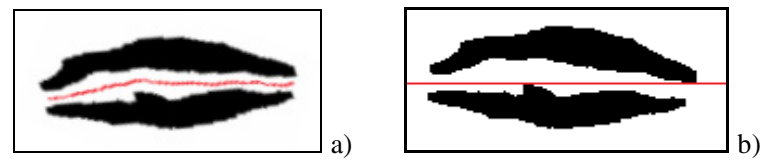

Fig. 2. Upper and lower lip separation line
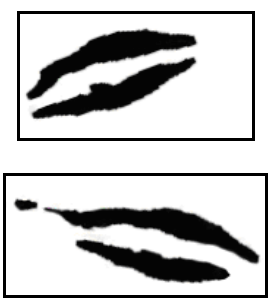

a)

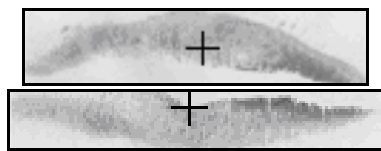

b)

c)

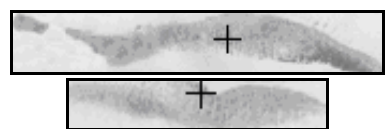

\section{,}


mask is placed outside the source lip print image $I$. To calculate the value of the central point of the mask, here marked with a cross, the values of the pixels of the original image I covered by the mask are convolved with the mask's elements.

Let the eight smoothing masks be denoted as $S^{J}$, where $J=1, \ldots, 8$. Then, for each position of the mask $S^{J}$, the value of its central pixel can be calculated:

$$
p^{J}=\sum_{a=0}^{4} \sum_{b=0}^{4} I(x+a-2, y+b-2) \cdot S^{J}(a, b) .
$$

Because the area of the mask $S^{J}$ must always be located entirely inside the image $I$, the procedure (4) begins at the point $x=y=2$ of image $I$. For each pixel, the values $p^{J}$ of the mask $J$ are successively calculated via the convolution of each mask with the image $I$. For each pixel, the mask $S^{b}$ with the largest cumulative value is selected:

$$
b=\underset{J}{\arg \max }\left\{p^{J}, J=1, \ldots, 8\right\}
$$

The value $p^{b} / 5$ now replaces the appropriate element of the image $I(x, y)$.

The convolution procedure (4) is repeated for each pixel of the image $I$ over the range $x \in[2, M-2]$ and $y \in[2, N-2]$. The effect of the image smoothing within the region of interest is shown in Fig. 5b. To compare the results obtained, fragments of the selected images are shown both before and after the smoothing procedure.
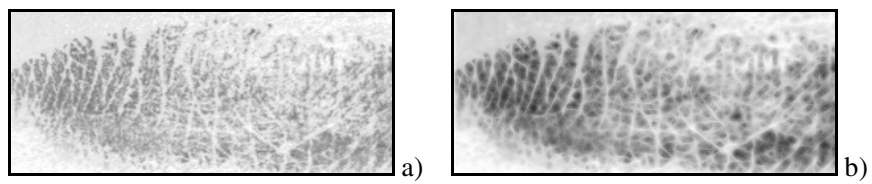

Fig. 5. The result of lip prints smoothing: a) a fragment of a lip print before smoothing, b) the same fragment after smoothing

In the second stage, the top-hat transformation is applied to the lip print image. This transformation extracts highlights (the small elements and details in a given image). In practice, the image is processed by each structural element and the processed image then subtracted from the original image. In the proposed approach, flat, diskshaped structural elements were applied [9]. The purpose of this procedure is to emphasize the lines of the lip pattern and to separate them from their background. To increase the effectiveness of this algorithm, this transformation was applied twice using two different sizes of structural masks. Two masks were used: a $5 \times 5$ mask to highlight thin lines (of up to 3 pixels); and an $11 \times 11$ mask to highlight the thick lines (of more than 3 pixels). The effects of these operations are depicted in Fig. 6a and 6b. After the top-hat (T-H) transformation, the image $I^{T-H}$ is obtained.

In the subsequent stage of the lip pattern extraction process, the image is converted into a binary representation. This procedure involves the application of a formula (6)to each of the two images resulting from the top-hat transformation. For the image 

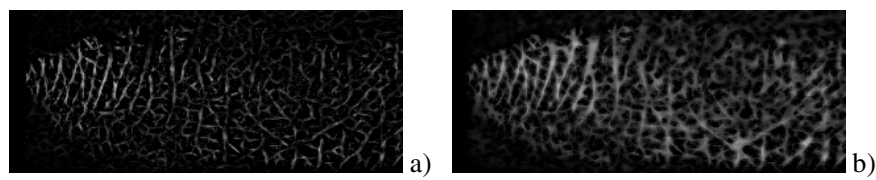

Fig. 6. Fragment of the lip print after the top-hat transformation: a) the thin lines emphasised (the $5 \times 5$ mask), b) the thick lines emphasised (the $11 \times 11$ mask)

with the thin lines emphasised, the conversion threshold value was set to be $t=15$, while for the thick line image this parameter became $t=100$.

$$
I_{B I N}(x, y)=\left\{\begin{array}{lll}
1 & \text { for } & I^{T-H}(x, y)>t \\
0 & \text { for } & I^{T-H}(x, y) \leq t
\end{array} .\right.
$$

The effect of the conversion of the lip print image into a binary representation is seen in Fig. 7.
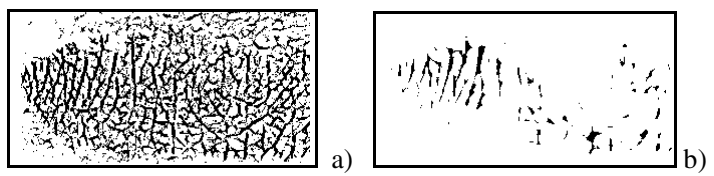

Fig. 7. Fragment of the lip print converted into a binary representation: (a) the thin lines emphasised (threshold of 15), (b) the thick lines emphasised (threshold of 100)

In the last stage, the sub-images of the thin and thick lines are combined into one single image, and this unified image is de-noised. For the noise reduction, appropriate $7 \times 7$ dimensional masks have been designed. Each of the 20 different masks is depicted in Fig. 8. Each mask, $M_{J}$, where $J=1, \ldots, 20$, consists of a different number of elements $e_{i}$ as indicated on Fig. 8. The two masks $M_{6}$ and $M_{11}$ consist of $e_{5}=e_{11}=5$ elements. Each of the other masks has 7 elements. Each element of each mask can take any value because these values are not important. Only the direction of the mask's elements needs to be considered. To simplify the mathematical formulas, the elements $e_{J}$ of each mask $M_{J}$ can take the value of 1 . Thus, both, the image $I_{B I N}$ and the masks $M_{J}$, have binary representation. Thus, each element of each mask $M_{J}$ nominates corresponding pixels from the source image $I_{B I N}$ to be counted.

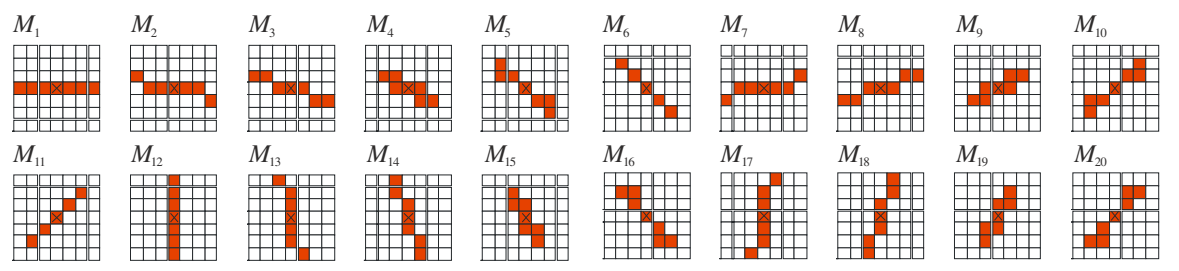

Fig. 8. Masks used for de-noising the binary image 
The $M_{J}$ masks are applied to each of the pixels of the source binary image $I_{B I N}$. Similarly to the previous masks, they are shifted from the left to the right and from the top of the lip print image $I_{B I N}$ downwards. For each given mask, the black pixels of the image $I_{B I N}$ that lie underneath the mask are counted. This process can be described more formally:

$$
p_{J}=\sum_{a=0}^{6} \sum_{b=0}^{6} I_{B I N}(x+a-3, y+b-3) \cdot M_{J}(a, b) .
$$

If the number $p_{J}$ of the black pixels of the image $I_{B I N}$ associated with the number of elements in the mask $M_{J}$ satisfies the condition $p_{J}<e_{J}$ then the analyzed pixel of the image $I_{B I N}(x, y)$ is converted to white. Otherwise the value of the pixel remains unchanged. The exemplary effects of this noise reduction method are shown in Fig. 9.

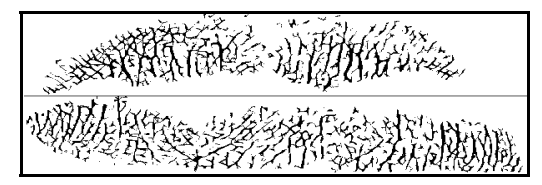

Fig. 9. Lip print image after the lip pattern extraction process

\section{The DTW-Based Comparison of Lip Imprints}

This process relies on a feature analysis and consists of determining the vertical, horizontal and diagonal projections of the lip pattern image. These projections create a special type of histograms.

Let the monochrome image $I$ of dimension $M \times N$, consist of pixels $p_{i}$ :

$$
I=\left\{p_{i}\right\}_{i=1}^{M \cdot N}, \text { where } p_{i} \equiv I(x, y) \in[0,1] .
$$

Let there be a set of projections:

A) $H_{c}^{0^{0}}=\operatorname{card}\left\{(x, y) \in I: I(x, y)=1 \wedge I(x, y) \in l_{c}\right\}$,

$$
l_{c}: y=c, c=1, \ldots, N \text {, }
$$

B) $H_{c}^{90^{0}}=\operatorname{card}\left\{(x, y) \in I: I(x, y)=1 \wedge I(x, y) \in l_{c}\right\}$,

$$
l_{c}: x=c, \quad c=1, \ldots, M
$$

C) $H_{c}^{+45^{0}}=\operatorname{card}\left\{(x, y) \in I: I(x, y)=1 \wedge I(x, y) \in l_{c}\right\}$,

$$
l_{c}: y=-x+c, \quad c=1, \ldots,\left\lfloor\sqrt{M^{2}+N^{2}}\right\rfloor,
$$

D) $H_{c}^{-45^{0}}=\operatorname{card}\left\{(x, y) \in I: I(x, y)=1 \wedge I(x, y) \in l_{c}\right\}$, 


$$
l_{c}: y=x+c, \quad c=1, \ldots,\left\lfloor\sqrt{M^{2}+N^{2}}\right\rfloor,
$$

where $l_{c}$ is an appropriate straight line equation.

The directional projections of the example lip print image are shown in Fig. 10.
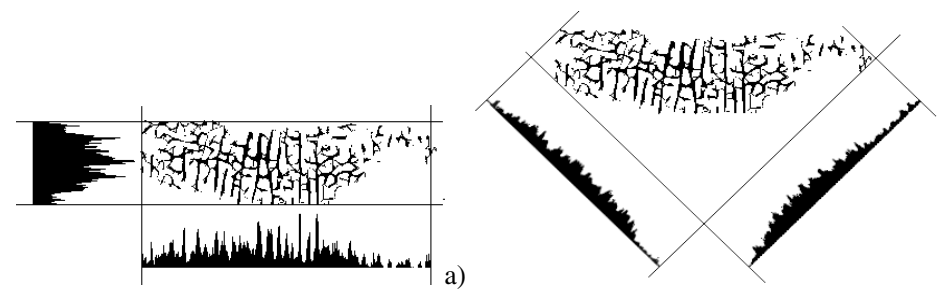

b)

Fig. 10. Projections (histograms) obtained from an example lip pattern: (a) vertical and horizontal projections, (b) diagonal projections at angles of $+45^{\circ}$ and $-45^{\circ}$

Each projection shows the number of black pixels that lie perpendicular to each axis direction: horizontal, vertical, and oblique at $\pm 45^{\circ}$. In order to evaluate the similarity between any two lip images, an appropriate measure of similarity needs to be defined. For image retrieval systems a variety of similarity measures and coefficients have been reported. It is known that the choice of similarity measure is related to the variability of the images within the same class. The method detailed in this paper demonstrates that similarity can be determined directly from the histograms produced. These histograms form discrete sequences composed of the histogram's elements $H_{c}^{a}$. Hence, the conformity of any two images can be determined by applying the DTW method. This approach was described in detail and successfully applied in a previous paper [7,9]. For the two images that are to be compared, first their histograms are generated. Each histogram can be treated as a series of numbers $\mathrm{N}_{+}$. In the first step, the local cost matrix $C$ of the alignment of the two sequences $A$ and $B$ is formed:

$$
C \in \mathrm{N}_{+}^{M \times N}: c_{i j}=\left|a_{i}-b_{j}\right| \text { and } a_{i} \in A, b_{i} \in B, i=1, \ldots, M, j=1 \ldots, N
$$

Many different alignment paths can be created over matrix's points, from $c_{11}$ to $c_{M N}$. The algorithm being summarized here finds the alignment path that runs through the low-cost areas of the cost matrix: see Fig. 11. The path with the minimal alignment cost will be referred to as the optimal alignment path. Let this path be denoted as $L$. Then:

$$
\operatorname{cost}(A, B)=\sum_{l=1}^{L} c_{i j}: c_{i j} \neq 0 .
$$

In other words, the similarity of two images can be defined as their appropriate histograms' matching cost. If the matching cost is lower, then the images are more similar. This procedure is performed for each type of projection (histogram) collected. For the 
two images being compared four costs values are computed. The costs obtained are then summarized and their average values calculated. The best DTW paths matching projections from two different sample lip prints are graphically depicted in Fig.11.
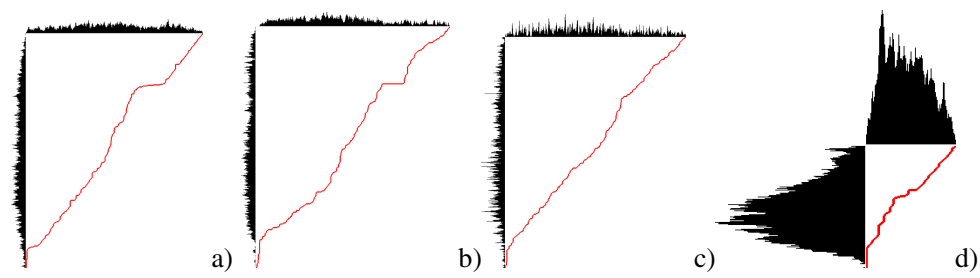

Fig. 11. Comparison of lip pattern projections using a DTW methodology: (a) oblique at $+45^{\circ}$, (b) oblique at $-45^{\circ}$, (c) horizontal, (d) vertical

This biometric recognition system has been tested in a closed set of trials by the evaluation of the CMC curves. In our case, for proposed database, ERR factor achieved value $\mathrm{EER}=\sim 21 \%$. The results obtained are presented in the chart of Fig. 12. Precise details of the methodology just described can be found in previously published work [9].

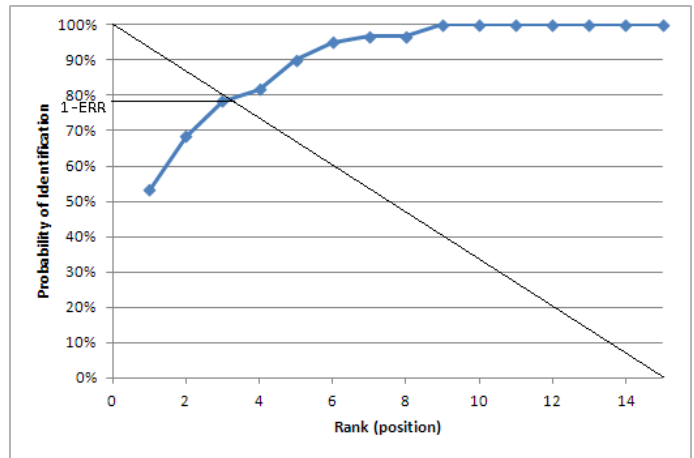

Fig. 12. The CMC curve with approximated 1-ERR point

It should be noted that lip recognition systems have not yet been properly and seriously investigated thus the literature on this topic is still very poor. For this reason, the results announced here must be regarded as good. Unfortunately, from a modern identification system's point of view, the recognition level achieved is still insufficient. For this reason, a modification of presented technique of image comparison has been introduced.

\subsection{Modification of the Basic Method}

Instead of summarizing and calculating the average matching cost of all collected histograms, each matching cost value can be individually analysed to build a rank list, which gives 8 rank lists for each lip print -4 for upper lip, and 4 for lower lip. In the new approach three methods of building rank lists were analyzed: 
- Class score is equal to the lowest (best) matching cost among class' samples.

- Class score is equal to the highest (worst) matching cost among class' samples.

- Class score is equal to the average matching cost of all class' samples.

According to other investigations reported $[13,14]$ it is possible to utilize vote counting methods for rank level fusion of biometric data, so the rank lists based on matching cost of each of the 8 histograms are used as a voting ballots to elect a Condorcet winner in a single round of voting performed using the Copeland's method as a vote counting algorithm.

\section{Basic Description of the Copeland Vote Counting Method.}

Let are 3 classifiers and 4 known objects $(A, B, C$ and $D)$, stored in the database. A new object $O_{\text {new }}$ should be classified. Each of the classifiers $C_{i}, i=1,2,3$ separately points out the list, sorted in descending order, of objects from the database according to their similarity $M_{i}=\left\{\operatorname{sim}\left(O_{\text {new }}, A\right), \ldots . . \operatorname{sim}\left(O_{\text {new }}, D\right)\right\}$ to the object $O_{\text {new }}$. This idea is presented on Fig. 13a. In the next stage classifiers return the sorted, with descending order, database objects which are most similar to the object $O_{\text {new }}$.

\begin{tabular}{|c|c|c|c|c|c|c|}
\hline Class & $M 1$ & $M 2$ & $M 3$ & $C 1$ & $C 2$ & $C 3$ \\
\hline A & 0,8 & 0,6 & 0,7 & $\mathrm{~A}$ & $\mathrm{~B}$ & $\mathrm{C}$ \\
\hline B & 0,6 & 0,8 & 0,4 & $\mathrm{~B}$ & $\mathrm{~A}$ & A \\
\hline C & 0,1 & 0,1 & 0,9 & $\mathrm{D}$ & $\mathrm{D}$ & B \\
\hline $\mathrm{D}$ & 0,5 & 0,2 & 0,1 & $\mathrm{C}$ & $\mathrm{C}$ & $\mathrm{D}$ \\
\hline
\end{tabular}

a)

\begin{tabular}{|c|c|c|c|}
\hline Pair & Winner & Looser & Ratio \\
\hline $\mathrm{A}, \mathrm{B}$ & $\mathrm{A}$ & $\mathrm{B}$ & $2: 1$ \\
\hline $\mathrm{A}, \mathrm{C}$ & $\mathrm{A}$ & $\mathrm{C}$ & $2: 1$ \\
\hline $\mathrm{A}, \mathrm{D}$ & $\mathrm{A}$ & $\mathrm{D}$ & $3: 0$ \\
\hline $\mathrm{B}, \mathrm{C}$ & $\mathrm{B}$ & $\mathrm{C}$ & $2: 1$ \\
\hline $\mathrm{B}, \mathrm{D}$ & $\mathrm{B}$ & $\mathrm{D}$ & $3: 0$ \\
\hline $\mathrm{C}, \mathrm{D}$ & $\mathrm{D}$ & $\mathrm{C}$ & $2: 1$ \\
\hline
\end{tabular}$\quad \checkmark$\begin{tabular}{|c|c|c|c|c|}
\hline Class & Victories & Defeats & Points \\
\hline $\mathrm{A}$ & 3 & 0 & 3 \\
\hline $\mathrm{B}$ & 2 & 1 & 1 \\
\hline $\mathrm{D}$ & 1 & 2 & -1 \\
\hline $\mathrm{C}$ & 0 & 3 & -3 \\
\hline
\end{tabular}

b)

Fig. 13. Principles of the rank list creation process (a) and pair-wise contest Condorcet's vote counting method (b)

In the next step a list of all possible pairs (combinations without repetitions) of classes is created (Fig. 13b). Class which has a higher position in most rank lists gains a point. Class which has a lower position looses a point. If there is a tie no points are granted. This, so-called pair wise contest, is illustrated on Fig. 13b. The last step is sorting the classes list according to points they gained in the pair-wise contest.

\section{Voting and Rank Technique}

As in previous case the tests were performed using the histograms and DTW method on a closed set of samples using CMC curves, and estimation of EER. Recognition accuracy of this method was significantly improved, Fig. 14 shows that the best results were achieved when voting and rank lists based on average matching cost across the class $(E R R=\sim 11,5 \%)$ were applied. The second best result was performed for voting on rank list based on a worst class representative (sample with highest matching cost across the class) with $E R R=\sim 13 \%$ and the worst results among voting 
trials were achieved when using samples with lowest matching cost as a class representatives $(E R R=\sim 17,5 \%)$ but it was anyways a better result than when ordering classes on a base of an average DTW matching cost.

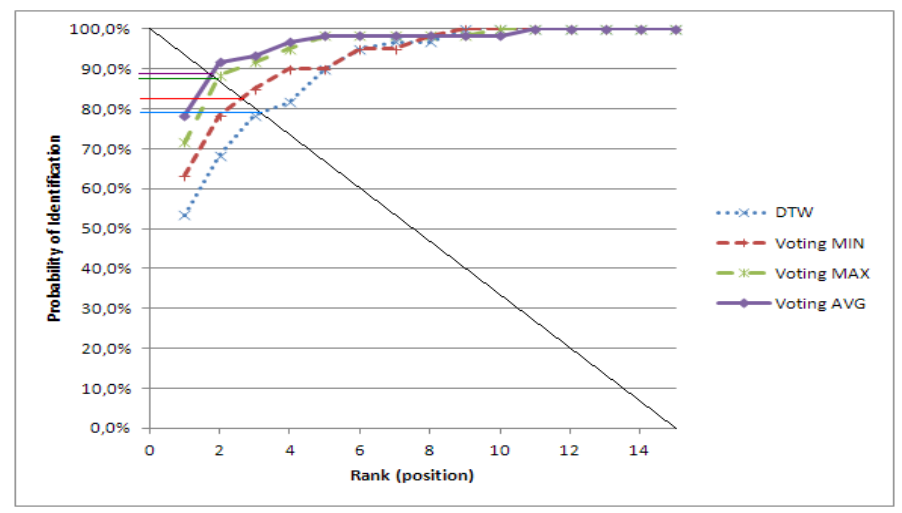

Fig. 14. CMC curves for modified algorithm

One thing worth noticing is the fact, that the basic DTW method and voting using best (minimal matching cost) class representatives have probability of identification of $100 \%$ at rank 9, when using voting with worst (maximal matching cost) class representative probability of identification of $100 \%$ is reached at rank 10 and when using class-average matching cost probability of identification of $100 \%$ is reached at rank 11.

\section{The Results Obtained}

A recognition technique measures some properties of an individual and stores that information as a template. This procedure is repeated for a number of individuals, and a database of templates is formed. We then need an identification technique to correctly discriminate between individuals. Two such techniques have been presented in this paper. In the study described here, 120 lip prints from 30 individuals (4 lip prints per person) were examined. These prints were entered into the database. Next, in a round-robin type procedure, one image was removed from the database and this extracted image was compared with all the remaining images in the database. From this dataset we used similarity scores ( 8 per one individual). As the voting results does not contain any score, just an order is important so it is not possible to introduce any threshold value to draw a ROC curves for identification in an open set environment, so the methods' efficiency was compared in a closed set environment using cumulative match characteristic curves (Fig. 14), where possibility of identification (PoI) and rank are defined as:

$$
P o I=\frac{T P}{T P+F P} \cdot 100 \% \text { and } \quad r a n k=F P-1
$$

where: $T P$ - true positive match and $F P$ - false positive match. 


\section{Conclusions}

The results of experiments demonstrate that presented here model of biometric system and a new kind of similarity scores give a good lip print recognition level. As similarity scores the DTW together with Copeland voting technique have been applied. The process for collecting lip prints was the same procedure as that used in forensic laboratories for collecting lip prints from suspects. First, latent lip prints were collected on paper using a cheiloscopic stamp. The visible lip prints were then scanned and converted into grayscale images with a resolution of 300 dpi. Proposed studies make it clear that if lip features are appropriately captured, the lip print might become an important tool for identification. For example, this procedure can be applied during forensic investigations (post mortems, at crime scenes, in relation to medicine, etc.).

\section{References}

1. Kasprzak, J., Leczynska, B.: Cheiloscopy. Human Identification on the Basis of a Lip Trace, pp. 11-29 (2001) (in Polish)

2. Sharma, P., Saxwna, S., Rathod, V.: Comparative reliability of cheiloscopy and palatoscopy in human identification. Indian Journal of Dental Research 20(4), 453-457 (2009)

3. Tsuchihashi, Y.: Studies on personal identification by means of lip prints. Forensic Science, 127-231 (1974)

4. Dougherty, E.: An introduction to morphological image processing (1992)

5. Rath, T.M., Manmatha, R.: Word image matching using dynamic time warping. In: IEEE Conference on Computer Vision and Pattern Recognition, pp. 521-527 (2003)

6. Smacki, L., Wrobel, K.: Lip Print Recognition Based on Mean Differences Similarity Measure. In: Burduk, R., Kurzyński, M., Woźniak, M., Żołnierek, A. (eds.) Computer Recognition Systems 4. AISC, vol. 95, pp. 41-49. Springer, Heidelberg (2011)

7. Smacki, L.: Lip traces recognition based on lines pattern. Journal of Medical Informatics and Technologies 15, 53-60 (2010)

8. Smacki, L., Porwik, P., Tomaszycki, K., Kwarcinska, S.: Lip print recognition using the Hough transform. Journal of Medical Informatics and Technologies 14, 31-38 (2010)

9. Smacki, L., Wrobel, K., Porwik, P.: Lip print recognition based on the DTW algorithm. In: Proc. of the Nature and Biologically Inspired Computing, Conf. Salamanca, Spain, pp. 601-606 (2011)

10. Utsuno, H., et al.: Preliminary study of post mortem identification using lip prints. Forensic Science International. 149(2-3), 129-132

11. Segui, M.A., et al.: Persistent lipsticks and their lip prints: new hidden evidence at the crime scene. Forensic Science International 112(1), 41-47 (2000)

12. Boole, R.M., et al.: Guide to biometrics. Springer Science+Buisnes Media Inc. (2004)

13. Kim, J.O., et al.: Lip print recognition for security systems by multi resolution architecture. Future Generation Computer Systems (20), 295-301 (2004)

14. Monwar, M., Gavrilova, M.L.: Robust Multimodal Biometric System using Markov Chain based Rank Level Fusion. In: VISAPP 2010 - Proceedings of the Fifth International Conference on Computer Vision Theory and Applications, Angers, France, vol. 2, pp. 458-463 (2010) 